\title{
Remuneração dos serviços de saúde bucal: formas e impactos na assistência
}

\author{
Different forms of payment systems \\ for dental services and their impact on care
}

\author{
Marina Lara Sória 1 \\ Ronaldo Bordin 2 \\ Luiz Cesar da Costa Filho 3
}

1 Programa de
Desenvolvimento da Gestão
em Saúde, Faculdade de
Administração, Universidade
Federal do Rio Grande do
Sul. Rua Ramiro Barcelos
2600, Porto Alegre, RS
90035-003, Brasil.
marinalarasoria@terra.com.br
2 Departamento de Medicina
Social, Faculdade de
Medicina, Universidade
Federal do Rio Grande do
Sul. Rua Ramiro Barcelos
2600, Porto Alegre, RS
90035-003, Brasil.
bordin@famed.ufrgs.br
3 Faculdade de Odontologia,
Pontifícia Universidade
Católica do Rio Grande
do Sul. Av. Ipiranga 6681,
prédio 6, Porto Alegre, RS
90619-900, Brasil.
periodontia@rocketmail.com

\begin{abstract}
The Brazilian dental care sector is facing a paradoxical crisis characterized by a surplus of dentists and a large contingent of people lacking dental care, thus highlighting the need to improve management strategies. One necessary step is to analyze the various payment schemes for dental services. This paper reviews two important approaches, fee for service and capitation, and considers the impacts and consequences of payment strategies on the dental care system.

Key words Dental Care; Oral Health; Dental Economics; Health Services

Resumo O setor odontológico está atravessando uma fase paradoxal, caracterizada pelo excesso de profissionais no mercado e pelo grande contingente populacional carente de tratamentos odontológicos. Com isso, há a necessidade crescente de serem aprimoradas habilidades gerenciais a fim de possibilitar a melhor organização do setor. Nesse contexto, o estudo das várias formas de remuneração dos serviços de saúde bucal assume papel relevante. Este artigo aborda duas importantes formas de pagamento (taxas por serviço e capitação) aos prestadores de serviço de saúde bucal, analisando impactos e conseqüências para o sistema de assistência odontológica.

Palavras-chave Assistência Odontológica; Saúde Bucal; Economia em Odontologia; Serviços de Saúde
\end{abstract}




\section{Introdução}

Atualmente, os gestores dos sistemas de saúde têm buscado maneiras de prover, em sua esfera administrativa, a atenção integral à saúde do indivíduo. Momento em que a saúde bucal, de reconhecida carência em nosso meio, assume papel relevante no planejamento das atividades multidisciplinares (Moysés \& Watt, 2000; Sheiham \& Moysés, 2000; Shinkai \& Cury, 2000).

Além disso, a última década foi rica na realização de estudos que enfatizam o fato de que a boca não deve ser considerada separadamente do organismo humano, e que mais importante do que as manifestações bucais de doenças sistêmicas são os impactos sistêmicos das doenças bucais (Rutkauskas, 2000; Williams \& Offenbacher, 2000). As relações entre saúde bucal e saúde geral são questões que precisam ser aproximadas, e a boca precisa ser conectada ao organismo (Damiano, 1993).

O setor odontológico brasileiro está atravessando uma fase paradoxal, na qual ocorre, simultaneamente, excesso de oferta e demanda por serviços odontológicos. Apesar do considerável declínio da prevalência de cárie no Brasil, sabe-se que grande parcela da população ainda sofre de problemas odontológicos, pois a redução dos índices de cárie ocorreu de forma heterogênea na população (Marcenes \& Bönecker, 2000; Nadanovsky, 2000; Pinto, 1999). O recente estudo da Fundação Instituto Brasileiro de Geografia e Estatística (IBGE) afirmou que $18,7 \%$ da população brasileira jamais tiveram a oportunidade de consultar-se com um cirurgião-dentista (Andrade, 2000). Por outro lado, ingressam no mercado de trabalho, anualmente, cerca de 12.000 profissionais que irão somar-se aos 160.718 já existentes, fazendo com que a população de cirurgiões-dentistas cresça cerca de 5,7\% ao ano, enquanto o crescimento anual da população brasileira é de 1,6\% (CFO, 2001; IBGE, 2001). Informações como estas apontam para a necessidade de se organizar o setor de forma a prover a assistência odontológica aos milhões de pessoas que dela necessitam e, ao mesmo tempo, absorver a abundância de profissionais no mercado de trabalho. Portanto, a habilidade gerencial, o conhecimento e compreensão das diferentes formas de organização dos sistemas de assistência e remuneração dos prestadores tornam-se, mais do que nunca, uma necessidade.

\section{Formas de remuneração dos prestadores em odontologia}

A prática odontológica tem-se tornado menos autônoma e mais complexa (Hernandez et al., 1999). A expansão das formas tripartites de assistência e a busca pela contenção dos custos crescentes pelas empresas do ramo originaram estudos e práticas de várias formas de pagamento aos prestadores de serviços de saúde (Guay, 1995; Hernandez et al., 1999; Médici, 1997). Além disso, sabe-se que o comportamento do cirurgião-dentista é influenciado pela forma de pagamento do serviço e que as conseqüências das formas de remuneração nos sistemas de assistência médica diferem daquelas encontradas nos sistemas de assistência odontológica (Palmer, 1993; Shenkin, 1999).

No Brasil, ainda são poucas as organizações de assistência odontológica que estão trabalhando com formas de pagamento alternativas às mais praticadas (taxas por serviço e salário). Entretanto, a compreensão deste tema é essencial para o gestor que objetiva promover atividades de saúde que produzam impacto com contenção de recursos.

As formas de remuneração mais comuns ao setor odontológico são: taxas por serviço, capitação, sistema de descontos programados, salário e reembolso direto.

A seguir, analisam-se duas modalidades de remuneração - taxas por serviço e capitação pelos seguintes enfoques: aspectos relacionados com o paciente, aspectos relacionados com o profissional e conseqüências para o sistema de assistência.

\section{Taxas por serviço}

A remuneração por taxas por serviço (fee-forservice), também conhecida como remuneração por ato ou por unidade de serviço, funciona com o auxílio de Tabelas pré-determinadas entre as partes, em que o comprador remunera o prestador pelo ato realizado (Beazoglou et al., 1988; Piola \& Vianna, 1995). É a forma de remuneração mais comum e mais praticada na área da saúde (Holloway, 1990). A remuneração de dentistas segundo os valores da Tabela Nacional de Convênios e Credenciamentos é um exemplo desta forma de pagamento.

O pagamento por taxas por serviço remunera o profissional pelo que já foi feito, sendo o chamado pós-pagamento por ato, já que o reembolso ocorre depois do tratamento concluído (Beazoglou et al., 1988; Riley, 1973). 


\section{a) Aspectos relacionados com o paciente}

Segundo Beazoglou et al. (1988), as várias formas de remuneração geram importantes conseqüências em relação ao controle de custos e efetividade de tratamento, mas não se pode subestimar a importância da satisfação do paciente. A relação entre a remuneração por serviço e a satisfação do paciente apresenta alguns pontos principais descritos a seguir.

- Maior liberdade de escolha do profissional pelo paciente, mesmo que seja dentro de uma rede definida (Christensen, 1994; Guay, 1995; Meskin, 1995).

- Interesses em comum, ou seja, o desejo do paciente é o mesmo do profissional, porque sempre quando ocorre a diminuição direta dos custos para o paciente sucede-se a uma maior procura por serviços; e o profissional também tem interesse em aumentar o número de procedimentos, uma vez que isso está diretamente ligado à sua lucratividade (Beazoglou et al., 1988). Com isso, o sistema de remuneração por taxas por serviço pode gerar um maior grau de comprometimento do dentista com o paciente (Mellor et al., 1990).

- Co-pagamento, posto que os planos que utilizam a taxa por serviço como forma de remuneração tendem a apresentar grande número de procedimentos que exigem a co-participação do paciente, situação que pode determinar a escolha do plano (Beazoglou et al., 1988). Vale lembrar que quanto maiores e mais freqüentes forem as taxas de co-participação, maior será o risco financeiro transferido ao paciente (Hernandez et al., 1999).

- O tratamento de doenças crônicas, segundo alguns estudos, tem apresentado melhores resultados dentro do sistema de taxas por serviço. Diante da natureza das principais doenças bucais, este aspecto pode ser particularmente relevante para o gestor dos sistemas de assistência odontológica (Shenkin, 1999).

- O acesso aos consultórios odontológicos pode ser mais fácil dentro do sistema de remuneração de taxas por serviço, pois pode haver uma distribuição geográfica que não exija do paciente investimento de tempo e dinheiro para locomoção pessoal. Este aspecto pode influenciar na escolha do plano odontológico (Beazoglou et al., 1988).

\section{b) Aspectos relacionados com o profissional}

Neste sistema, o tratamento realizado é a fonte de lucros do prestador (Figura 1), e não há risco financeiro para o mesmo, justamente o oposto do que ocorre no sistema de capitação (Guay,

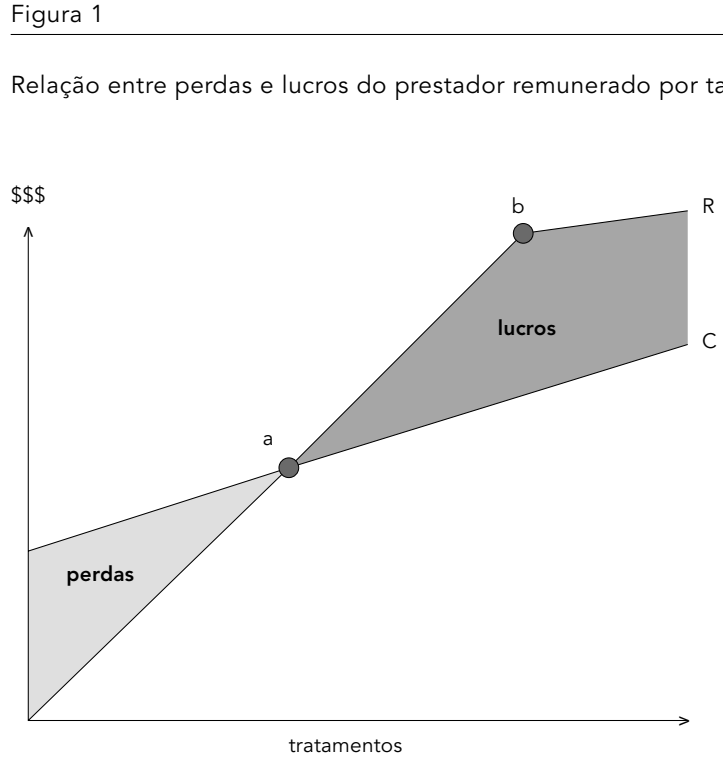

Fonte: Guay (1995).

1995; Hernandez et al., 1999; Shenkin, 1999). As maiores críticas ao sistema de pagamento por taxas por serviço estão relacionadas ao aumento de custos observados nos planos que utilizam este sistema (Beazoglou, et al., 1988). Segundo Médici (1997), quando o relacionamento entre os compradores e prestadores ocorre com base na remuneração de taxas por serviço, há a tendência de "crescimento desmesurado de custos".

A Figura 1 é composta por duas linhas: a linha " $\mathrm{R}$ " que representa o rendimento do consultório odontológico, e a linha "C" que expressa o custo deste estabelecimento. A linha dos custos "C" não inicia no ponto zero do gráfico, dado que sempre existem despesas fixas do consultório odontológico.

Analisando-se o gráfico, o ponto (a) representa o momento em que os rendimentos começam a superar os custos. À medida que aumenta o número de tratamentos feitos, aumenta também a despesa e o rendimento. $\mathrm{O}$ momento em que o rendimento supera os gastos (ponto "a"), é quando começa a existir lucro. Assim sendo, podemos visualizar de maneira clara que quanto maior o número de procedimentos realizados pelo cirurgião-dentista, maior será seu lucro. Unindo este fato com questões discutidas amplamente na literatura sobre o modelo de prática hegemônico na odontologia, como, por exemplo, o "Ciclo Restaurador Repetitivo” descrito por Elderton (1999) e com o já conhecido poder de indução de demanda 
inerente ao setor, podemos concluir que a remuneração por taxas por serviço gera riscos financeiros para o pagador do serviço.

O crescimento dos lucros de acordo com o número de tratamentos realizados não é ilimitado. O ponto (b) representa o momento em que pode ocorrer a diminuição da taxa de crescimento dos lucros, imposta pelos mecanismos de controle de consumo como, por exemplo, co-participação do paciente, estabelecimento de "tetos" de consumo, limitação do número de radiografias e outros, visto que a freqüência de co-pagamentos neste sistema costuma ser maior que no sistema de remuneração por capitação (Beazoglou et al., 1988; Marcus et al., 1995).

De acordo com a American Dental Association (ADA), os profissionais deveriam ser pagos pelo que eles fizeram e não pelo que vão fazer, ou seja, por intermédio de taxas por serviço ou reembolso direto (Riley, 1973).

No estudo de Mellor et al. (1990), os dentistas pagos por taxas por serviço queixaram-se de falta da liberdade para tratamentos e demonstraram maior procura por cursos de educação continuada para incrementar sua prática e acrescentar procedimentos na Tabela de remuneração. Neste estudo (que compara capitação com taxas por serviço), houve maior grau de comprometimento dos dentistas remunerados por taxas por serviço com seus pacientes. De uma forma geral, acredita-se que, dependendo dos valores da taxas, este tipo de remuneração pode ser bastante satisfatória para o profissional, que não está exposto a nenhum risco financeiro (Hernandez et al., 1999).

\section{c) Conseqüências para o sistema}

A forma de obtenção de lucros pelo prestador pode fazer com que ocorra o sobretratamento, vale dizer, o excesso do número de procedimentos diante da duvidosa necessidade de tratamento (Beazoglou et al., 1988; Friedman, 1996; Marcus et al., 1995; Riley, 1973; Shenkin, 1999). Em odontologia, esta característica pode ser particularmente perigosa, posto que é comum os profissionais terem dificuldade em separar o tratamento necessário do que pode ser feito, fruto do modelo de prática tecnicista curativista já descrito anteriormente (Friedman, 1996). Outro agravante é a variabilidade de diagnóstico, muito comum entre os profissionais de odontologia (Ekstrand, 2000), fato que dificulta as pesquisas que buscam avaliar o sobretratamento gerado pelo sistema de pagamento por taxas por serviço.
Mesmo assim, foram constatadas as seguintes tendências neste sistema de remuneração: - encorajamento para procedimentos de alto custo e alta complexidade, desestimulando a prevenção (Grant, 1991; Holloway et al., 1990; Lennon et al., 1990; Mellor et al., 1990; Riley, 1973);

- dificuldade de controle e previsibilidade de custos (Médici, 1997; Pinto, 2000);

- melhores resultados para tratamento de doenças crônicas (Shenkin, 1999);

- encorajamento à desonestidade ao relatar os procedimentos realizados (Christensen, 1994; Piola \& Vianna, 1995);

- a elaboração do plano de tratamento pode ser guiada pelos valores das taxas, mais do que pela necessidade de tratamento do paciente (Shenkin, 1999);

- dificuldade de controle de qualidade e possível comprometimento da mesma (Christensen, 1994; Marcus et al., 1995; Shenkin, 1999); e - consumo de tempo no preenchimento de papéis administrativos, uma vez que é exigida a descrição detalhada do tratamento realizado.

Muitos autores enfatizam que a remuneração por meio de taxas por serviço é, teoricamente, desestimuladora das práticas preventivas e agrava o poder de indução de demanda (Grant, 1991; Piola \& Vianna, 1995; Riley, 1973; Shenkin, 1999). De fato, os estudos realizados na Grã-Bretanha por Holloway et al., (1990), que compararam a performance dos dentistas remunerados por capitação com os por taxas por serviço, constataram que os profissionais pagos por ato preocuparam-se menos com prevenção e educação de seus pacientes. Por outro lado, Beazoglou et al. (1988), em seu estudo também de natureza comparativa, constaram o inverso: os dentistas remunerados por ato realizaram maior número de procedimentos preventivos; o achado foi atribuído ao fato de que os dentistas do sistema de capitação eram pressionados por seus pacientes a realizarem tratamentos de ortodontia e prótese (devido às menores taxas de co-participação e grande interesse da população), fazendo com que o tempo fosse consumido com estes serviços.

Pinto (2000:43), ao descrever o sistema de remuneração por taxas por serviço, enfatizou: "O regime de remuneração por ato não é recomendável por tender ao aumento dos custos, dificultar a previsibilidade dos gastos e ser praticamente incontrolável. Para a categoria odontológica pode ser danoso, pois condiciona o tratamento à remuneração criando problemas éticos por vezes insuperáveis".

Os gestores dos sistemas que adotam o modelo de pagamento por taxas por serviço po- 
dem controlar os custos pela limitação do tamanho da rede credenciada, posto que cada profissional tem capacidade limitada de trabalho. Além disso, sabe-se que o sobretratamento, que pode estar presente neste modelo, não é infinito, podendo ser regulado pelas forças de mercado e pelos valores éticos na relação entre profissional e paciente (Beazoglou et al., 1988).

\section{Capitação}

Estima-se que cerca de $4 \%$ da população norte-americana sejam beneficiários de planos que utilizam a capitação como modelo de pagamento a seus prestadores, e 13\% dos dentistas (clínicos-gerais) são remunerados desta forma (Pride, 1991).

O sistema de capitação funciona com base no pagamento de um valor mensal fixo per capita, isto é, o profissional recebe antecipadamente uma determinada quantia por cada paciente que compõe sua rede, independente do tratamento que será realizado (Holloway et al., 1990; Piola \& Vianna, 1995). Este modelo é prépago, a saber, o prestador é remunerado pelos serviços que ainda vai fazer (Beazoglou et al., 1988; Riley, 1973).

\section{a) Aspectos relacionados com o paciente}

Os pontos do modelo de capitação ligados à satisfação do paciente são descritos a seguir.

- Conflito de interesses entre profissional e paciente, posto que o paciente tem o desejo de fazer vários tratamentos (a redução de custos diretos estimula a demanda), e o dentista tem o interesse de fazer apenas o mínimo necessário (Beazoglou et al., 1988; Riley, 1973; Shenkin, 1999).

- A liberdade de escolha do cirurgião-dentista pelo paciente fica reduzida, quando comparada a outros modelos (Christensen, 1994; Guay, 1995; Meskin, 1995).

- A locomoção até o consultório do dentista pode ser mais dispendiosa, quando comparada àquela dos planos de taxas por serviço, pois o sistema de capitação possui uma rede reduzida de profissionais credenciados (Beazoglou et al., 1988).

- As taxas de co-participação tendem a ser bastante reduzidas nos planos de capitação, fato favorável ao paciente, podendo determinar a escolha do plano. Nos Estados Unidos os serviços de ortodontia e prótese apresentam menores taxas de co-participação, quando comparadas às de outros planos. Alguns autores acreditam que esta é a razão para o crescimento deste modelo de pagamento neste país (Beazoglou et al., 1988; Friedman, 1996).

- Pode acontecer a situação de escolha de pacientes pelo profissional, que, objetivando o controle de seus custos, priorizará o atendimento a pacientes jovens e de baixo risco (Guay, 1995).

- Os profissionais podem utilizar mecanismos para reduzir o número de tratamentos pela limitação do acesso, exacerbando o tempo de espera no consultório e dificultando a marcação de consultas (Marcus et al., 1995).

\section{b) Aspectos relacionados com o profissional}

O risco financeiro do sistema de capitação é transferido ao prestador de serviços, fato que determina o maior controle de custos do comprador. Neste sentido, o pagamento por capitação é oposto ao modelo de taxas por serviço, visto que o tratamento prestado é o centro dos gastos, drenando os lucros do prestador (Figura 2) (Guay, 1995; Herandez et al., 1999; Riley, 1973; Shenkin, 1999).

Analisando-se a Figura 2, tem-se a linha "R", que expressa o rendimento do consultório odontológico, e as linhas "C1" e "C2", que representam os custos em diferentes situações. As linhas dos custos iniciam-se acima do ponto zero, devido às despesas fixas do consultório, já mencionadas anteriormente. A linha que

Figura 2

Relação entre perdas e lucros do prestador remunerado por capitação.

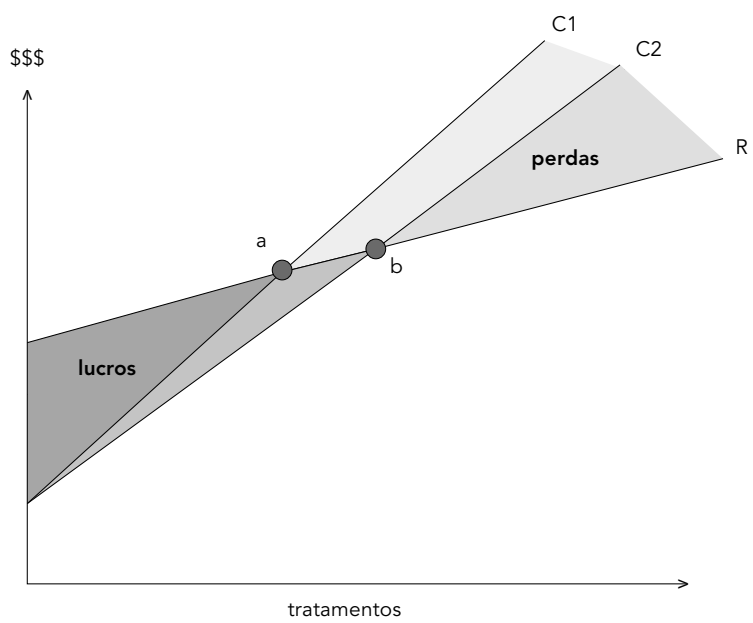

Fonte: Guay (1995). 
representa o rendimento também não se inicia no ponto zero, pois o modelo de pagamento da capitação prevê um valor fixo de acordo com o número de pacientes assistidos pelo profissional. A existência de taxas de co-pagamento e eventuais serviços não cobertos pelo plano produz a ligeira inclinação observada na linha "R". A linha "C1" ilustra a situação na qual o profissional prioriza procedimentos curativos, mais caros e demorados. Nesta situação, o lucro do profissional diminui à medida que ele efetua maior número de procedimentos, até o momento em que as despesas tornam-se maiores que o lucro (ponto “a”). A linha "C2" mostra que, quando o dentista prioriza atividades educativas e preventivas, pode aumentar o número de procedimentos, aprimorar o acesso e demorar mais para perder a lucratividade de seu consultório, momento representado pelo ponto (b) (Guay, 1995).

Alguns dentistas norte-americanos estão assustados com a expansão deste modelo de pagamento nos Estados Unidos, podendo-se perceber a coexistência de grupos que defendem e atacam este modelo. Os principais pontos destacados são mencionados a seguir:

- Os profissionais alegam que o modelo de capitação prioriza o controle de custos e não a qualidade de tratamento; defendem que o modelo não representa os interesses do paciente, interferindo na relação profissional-paciente, pois os dentistas têm que encontrar formas de controlar o fluxo de procedimentos sem perder seu paciente (Beazoglou et al., 1988; Pride, 1991; Shenkin, 1999);

- A capitação pode ser uma boa fonte de renda aos novos profissionais que não possuem toda a agenda do consultório preenchida (Pride, 1991), fato que é muito comum no Brasil;

- Os profissionais não gostam de assumir o risco financeiro da situação porque isto lhes exige maior habilidade administrativa e empreendedora (Hernandez et al., 1999; Pride, 1991; Riley, 1973);

- O dentista pago por capitação comporta-se como segurador, isto é, ele deve diluir o risco por meio do aumento do número de pacientes, e uma rede pequena de beneficiários é inviável (Guay, 1995; Hernandez et al., 1999);

- Os planos permitem o acesso à saúde para populações que não teriam condições de arcar diretamente com o custo do tratamento odontológico (Christensen, 1994; Pride, 1991);

- Os estudos de Holloway et al. (1990), Lennon et al. (1990) e Mellor et al. (1990), que avaliaram a visão dos profissionais remunerados pelos sistemas de capitação e taxas por serviço, constataram que aqueles pagos por capitação relataram sentir maior liberdade clínica no tratamento de seus pacientes. De fato, não há a necessidade de encaixar todo procedimento realizado dentro de um padrão de Tabela definido e é possível maior flexibilidade dos recursos (Schreiner, 2000);

- Apesar da transferência do risco financeiro ao prestador, a capitação oferece segurança financeira ao profissional, uma vez que lhe garante renda fixa mensal (Guay, 1995; Holloway et al., 1990);

- Há estímulo de responsabilidade do profissional para com seu paciente (Holloway et al., 1990);

- O tempo gasto com preenchimento de papéis e descrição de procedimentos realizados é menor, o que é favorável a dentistas e a pacientes (Holloway et al., 1990).

\section{c) Conseqüências para o sistema}

Vários autores enfatizam que o sistema de capitação pode gerar o subtratamento, ou seja, a não-satisfação das necessidades de tratamento, justamente por funcionar de maneira oposta ao modelo de taxas por serviço. Há motivos racionais para que o profissional deixe de prestar o atendimento necessário, pois assim ele teria maior lucratividade. Todavia, existem limites como, por exemplo, a saída do paciente da rede de beneficiários do profissional, e aspectos éticos da relação profissional/paciente (Beazoglou et al., 1988; Hernandez et al., 1999; Holloway et al., 1990; Marcus et al., 1995; Riley, 1973; Shenkin, 1999). Além disso, Holloway et al. (1990), em seu estudo comparativo, não encontraram evidência de negligência no tratamento odontológico dos pacientes pertencentes ao plano de capitação. Da mesma forma, alguns estudos comprovaram que os pacientes de planos de capitação apresentam o mesmo padrão de saúde bucal dos beneficiários de planos que remuneram o profissional por taxas por serviço (Friedman, 1996; Shenkin, 1999). Acrescente-se que a variabilidade de diagnósticos e a dificuldade dos profissionais em distinguir entre o tratamento essencial e o possível de ser feito (Friedman, 1996) levam a crer que mais estudos sobre a ocorrência de subtratamento são necessários; sabe-se também que existem diversos fatores que podem contribuir, ou não, para a ocorrência desta característica do modelo de capitação como, por exemplo, o perfil epidemiológico da população assistida, o valor da taxa mensal per capita e o grau de procura dos pacientes (Guay, 1995).

Alguns autores alegam que os dentistas pagos por capitação tendem a tratar as lesões de 
cárie mais tardiamente que seus colegas remunerados por taxas por serviço. Este comportamento, de acordo com o contexto, pode ser avaliado como uma tendência a priorizar a prevenção e controle da doença, ou como negligência no tratamento (Holloway et al., 1990, Marcus et al., 1995). O fato é que conclusões precipitadas não podem ser tomadas, e a análise de um fator isolado não pode ser fonte de tomada de decisões do gestor (Beazoglou et al., 1988).

Contudo, algumas conseqüências da capitação são claras:

- Ocorre a tendência do profissional em realizar tratamentos simples e de custos reduzidos, evitando resolução de casos complexos e dispendiosos (Holloway et al., 1990; Marcus et al., 1995; Mellor et al., 1990; Shenkin, 1999);

- Há o encorajamento da prevenção e incentivo financeiro para contenção de recursos (Holloway et al., 1990; Lennon et al., 1990; Piola \& Vianna, 1995; Shenkin, 1999);

- Os planos de capitação, devido às baixas taxas de co-participação, podem apresentar consumo excessivo de serviços, gerando prejuízos ao profissional (Marcus et al., 1995, Hernandez et al., 1999, Holloway et al., 1990);

- A remuneração por capitação é indicada para planos de assistência odontológica a crianças, pois além do tratamento curativo infantil ser mais simples e barato, a prevenção em crianças apresenta melhores, mais rápidos e duradouros resultados quando comparados com àqueles encontrados em adultos (Holloway et al., 1990; Shenkin, 1999).

Do ponto de vista do gestor, o sistema de capitação não gera riscos financeiros à empresa, sendo recomendado para controle e planejamento de despesas (Guay, 1995; Hernandez et al., 1999; Riley, 1973; Shenkin, 1999).

Vale lembrar que o perfil epidemiológico da população a ser assistida pelo plano é um relevante fator a ser considerado pelo gestor, no momento em que este for decidir pela forma de remuneração de seus prestadores. Estudos têm demonstrado que o atendimento a crianças e grupos com pouca necessidade de tratamento acumulada pode gerar melhor resultado caso seja inserido no sistema de remuneração por capitação (Pride, 1991).

A questão da qualidade da assistência é descrita como ruim pelos profissionais que são contra o modelo de capitação (Christensen, 1994; Matsuishi, 1992). No entanto, aqueles que defendem este sistema enfatizam que a qualidade da assistência odontológica é dependente de vários fatores, entre eles, a própria consciência do dentista (Grant, 1991; Marcus et al.,
1995; Pride, 1991). Além disso, a qualidade em saúde está relacionada a diversos aspectos da assistência, não devendo ser avaliada valendose da análise de um fator isolado (Silver, 1992).

Nos Estados Unidos, onde o pagamento por capitação está em expansão, os planos odontológicos que utilizam este modelo estão demonstrando a tendência de reduzir a taxa fixa paga ao prestador e aumentar o número e valor das taxas de co-participação. O gestor que optar pelo pagamento de seus prestadores pela capitação deve saber que o excesso de co-participação é inconsistente com a filosofia do sistema, gerando o desestímulo para realização de procedimentos básicos e preventivos (Friedman, 1996). Segundo Marcus et al. (1995), as principais premissas do modelo de remuneração por capitação são:

- estímulo de uso regular dos serviços de saúde;

- controle e limitação de exclusões, fatores moderadores e co-pagamentos; e

- remuneração do profissional suficiente para cobrir os serviços prestados.

A forma de remuneração dos prestadores de serviços de saúde pode alterar o perfil epidemiológico da população assistida. Como exemplo, o caso da Nova Zelândia, em que o número médio de restaurações por criança era de 4,4, e foi reduzido para 1,5 em 10 anos (1970-1980). A mudança do modelo de pagamento foi apontada como o fator crucial para a alteração da filosofia de tratamento dos profissionais daquele país (Marcenes \& Bönecker, 2000).

Síntese de incentivos proporcionados pelas formas de remuneração

Conforme já descrito, as várias formas de remuneração produzem distintos efeitos no comportamento do prestador de serviços de saúde, interferindo diretamente na gestão dos sistemas de assistência e na prática da odontologia. Com isso, é importante que cada gestor possa identificar, implantar e gerenciar a modalidade de pagamento que mais se adapte às características de seu serviço de saúde.

A Tabela 1 estabelece uma comparação entre as formas de remuneração no que diz respeito ao comportamento do prestador e às conseqüências para o sistema de assistência.

\section{Considerações finais}

O contexto atual exige que gestores de sistemas de saúde, públicos e privados, detenham conhecimentos além das tradicionais ferramentas gerenciais. É necessária a compreensão dos 
Tabela 1

Síntese de incentivos proporcionados por duas formas de remuneração dos serviços de saúde bucal.

\begin{tabular}{|c|c|c|c|c|}
\hline Método & $\begin{array}{l}\text { Número de } \\
\text { pacientes }\end{array}$ & $\begin{array}{l}\text { Número de atos } \\
\text { por consulta }\end{array}$ & Gastos globais & $\begin{array}{l}\text { Risco financeiro } \\
\text { para o profissional }\end{array}$ \\
\hline Taxas por serviço & $\bullet$ & $\bullet$ & $\bullet$ & - \\
\hline Capitação & $\bullet$ & - & - & $\bullet$ \\
\hline
\end{tabular}

Fonte: Pinto (2000).

Símbolos:

- = Incentivo para produzir mais ou para aumentar gastos globais; apresenta risco financeiro ao prestador

- = Incentivo para produzir menos ou reduzir gastos globais; não apresenta risco financeiro ao prestador.

mecanismos básicos que determinam a doença na população, já que o objeto de trabalho do setor é a saúde. Com isso, a abordagem das principais formas de remuneração praticadas no setor demonstrou que uma mesma forma de pagamento pode gerar conseqüências dis- tintas, dependendo do setor da saúde em que se aplica, fazendo com que este tema deva ser cuidadosamente avaliado pelos gestores que buscam alcançar maiores níveis de efetividade e eficiência em organizações de assistência odontológica.

\section{Referências}

ANDRADE, M., 2000. Sem-dentistas. Revista da Associação Brasileira de Odontologia, 8:137-138.

BEAZOGLOU, T. J.; GUAY, A. H. \& HEFFLEY, D. R. 1988. Capitation and fee-for-service dental benefit plans: Economic incentives, utilization, and service-mix. Journal of the American Dental Association, 116:483-487.

CHRISTENSEN, G. J., 1994. How should dental bills be paid? Journal of the American Dental Association, 125:1013-1014.

CFO (Conselho Federal de Odontologia), 2001. Números do CFO. 18 Abril 2001 <http://www.cfo.org.br>.

DAMIANO, P. C., 1993. The Iowa Oral Health Care Reform Workshop. Journal of Dental Education, 57:923-924.

EKSTRAND, K., 2000. Diagnóstico de cárie. In: Promoção de Saúde Bucal na Clínica Odontológica (Y. P. Buichi, org.), pp. 125-148, São Paulo: Editora Artes Médicas.

ELDERTON, R. J., 1999. O ciclo restaurador repetitivo. In: Promoção de Saúde Bucal (L. Kriger, org.), pp. 195-200, São Paulo: Editora Artes Médicas. 
FRIEDMAN, J. W., 1996. Capitation in dentistry and appropriate dental care. Journal of Public Health Dentistry, 56:306-308.

GRANT, A. M., 1991. Capitation. Journal of the American Dental Association, 122:12.

GUAY, A. H., 1995. Understanding managed care. Journal of the American Dental Association, 126:425433.

HERNANDEZ, J. B.; COULTER, I.; GOLDMAN, D.; FREED, J. \& MARCUS, M., 1999. Managed care in dental markets: Is the experience of medicine relevant? Journal of Public Health Dentistry, 59:2432.

HOLLOWAY, P. J.; LENNON, M. A.; MELLOR, A. C.; CONVENTRY, P. \& WORTHINGTON, H. V., 1990. The capitation study: Does capitation encourage 'supervised neglect'? British Dental Journal, 168: 119-121.

IBGE (Fundação Instituto Brasileiro de Geografia e Estatística), 2001. Censo 2000. 20 Fevereiro 2001 <http://www.ibge.gov.br>.

LENNON, M. A.; WORTHINGTON, H. V.; CONVENTRY, P.; MELLOR, A. C. \& HOLLOWAY, P. J., 1990. The capitation study: Does capitation encourage more prevention? British Dental Journal, 168:213215.

MARCENES, W. \& BÖNECKER, M. J. S., 2000. Aspectos epidemiológicos e sociais das doenças bucais. In: Promoção de Saúde Bucal na Clínica Odontológica (Y. P. Buichi, org.), pp. 76-98, São Paulo: Editora Artes Médicas.

MARCUS, M.; COULTER, I. D.; FREED, J. R.; ATCHINSON, K. A.; GERSHEN, J. A. \& SPOLSKY, V. W., 1995. Managed care and dentistry: Promises and problems. Journal of the American Dental Association, 126:439-446.

MATSUICHI, R. K., 1992. Fee-for-service. Journal of the American Dental Association, 123:10.

MÉDICI, A. C., 1997. O plano Clinton e a cultura do pluralismo estruturado. In: A Economia Política das Reformas em Saúde (A. C. Médici, org. ), pp. 29-39, Porto Alegre: Instituto de Administração Hospitalar e Ciências da Saúde.

MELLOR, A. C.; COVENTRY, P.; WORTHINGTON, H. V.; HOLLOWAY, P. J. \& LENNORN, M. A., 1990. The capitation study: The views of participating dentists and the profession. British Dental Journal, 168:303-305.

MESKIN, L. H., 1995. If two's company, three may be a crowd. Journal of the American Dental Association, 126:402-406.

MOYSÉS, S. T. \& WATT, R., 2000. Promoção de saúde bucal - definições. In: Promoção de Saúde Bucal na Clínica Odontológica (Y. P. Buischi, org.), pp. 1-22, São Paulo: Editora Artes Médicas.
NADANOVSKY, P., 2000. O declínio da cárie. In: Saúde Bucal Coletiva (V. G. Pinto, org.), pp. 341-349, 4a Ed. São Paulo: Editora Santos.

PALMER, C., 1993. Dentistry and medicine: How they differ and why that should make a difference in health system reform. Journal of the American Dental Association, 124:42-43.

PINTO, V. G., 1999. Epidemiologia das doenças bucais no Brasil. In: Promoção de Saúde Bucal (L. Kriger, org.), pp. 27-42, 2a Ed. São Paulo: Editora Artes Médicas.

PINTO, V. G., 2000. Saúde Bucal Coletiva. 4ạ Ed. São Paulo: Editora Santos.

PIOLA, S. F. \& VIANNA, S. M., 1995. Formas de financiamento dos gastos com saúde. In: Economia da Saúde: Conceito e Contribuição para a Gestão da Saúde (S. F. Piola \& S. M. Vianna, org.), pp. 31-41, Brasília: Instituto de Pesquisa Econômica Aplicada.

PRIDE, J., 1991. Capitation: The choice is yours. Journal of the American Dental Association, 122:78-80.

RILEY, L. C., 1973. Disadvantages of HMOs and capitation. Journal of the Californian Dental Association, 1:40-47.

RUTKAUSKAS, J. S., 2000. The medical necessity of periodontal care. Periodontology 2000, 23:151-156.

SCHREINER, M., 2000. Modelos de Pagamento de Prestadores de Serviços de Saúde. Monografia de Especialização, Porto Alegre: Escola de Administração, Universidade Federal do Rio Grande do Sul.

SHEIHAM, A. \& MOYSÉS, S. J., 2000. O papel dos profissionais de saúde bucal na promoção de saúde. In: Promoção de Saúde Bucal na Clínica Odontológica (Y. P. Buischi, org.), pp. 23-38, São Paulo: Editora Artes Médicas.

SHENKIN, J., 1999. The future of dental managed care in the US. Health Policy, 47:225-239.

SHINKAI, R. S. A. \& CURY, A. D. B., 2000. O papel da odontologia na equipe interdisciplinar: Contribuindo para a atenção ao idoso. Cadernos de Saúde Pública, 16:1099-1109.

SILVER, L., 1992. Aspectos metodológicos em avaliação dos serviços de Saúde. In: Planejamento Criativo: Novos Desafios em Políticas de Saúde (E. Gallo, F. Rivetta \& M. H. Machado, org.), pp. 195-210, Rio de Janeiro: Relume Dumará.

WILLIAMS, R. C. \& OFFENBACHER, S., 2000. Periodontal medicine: The emergence of a new branch of periodontology. Periodontology 2000, 23:9-12.

Recebido em 23 de abril de 2001

Versão final reapresentada em 9 de novembro de 2001 Aprovado em 22 de fevereiro de 2002 